\title{
Fixed Progesterone-primed ovarian stimulation in polycystic ovarian syndrome: RCT A.E.Tahoun ${ }^{1}$, M.A.Elgazzar ${ }^{2}$, A.A.Shedid ${ }^{2}$, and A.Y.Rezk ${ }^{2}$ \\ 1 obstetrics and gynecology, Alsaadieen hospital, Ministry of health, Egypt \\ ${ }^{2}$ obstetrics and gynecology, Dept.,Faculty of Medicine, Benha Univ., Benha, Egypt \\ E-mail:asmaatahoun80@gmail.com
}

\begin{abstract}
Background: In vitro fertilization for women with polycystic ovarian syndrome is an essential (PCOS). New methods to improve clinical outcomes are still needed. Objective: this research investigated the effectiveness of PPOS and compared it with the standard PCOS antagonist regimen. Materials and procedures: A total of 76 PCOS women eligible for aided therapy of reproductive technology were recruited in this RCT from February 2020 to May 2021 and put in random two groups (n=38/per group). Since the sixth day of the cycle, the PPOS group has received $20 \mathrm{mg} /$ day orally of Dydrogesterone, while the control group received antagonist treatment. In addition to safety, our results were chemical and clinical pregnancy. Results: oocyte retrieval counts, oocyte metaphase II (MII) and MI have been comparable. There are also comparable numbers of fertilized oocytes and cleaved embryos without any statistical difference in the number of injected oocytes. Lower antagonistic gonadotropin dosage than the PPOS group (2957.8 \pm 301.9 vs 3197.4 \pm 545.9$)$, with higher stimulation times respectively in the PPOS group than the antagonist. Similar findings across both main outcome groups (OHSS) with no instances of FPPOS and just one case (2.6 percent) of moderate OHSS antagonists. Similar findings also for secondary results (biochemical pregnancy rate , clinical pregnancy rate and miscarriage rate). Conclusion: the established In PCOS patients receiving IVF/ICSI therapy, PPOS is a safe and successful procedure.
\end{abstract}

Key words: Progesterone, Polycystic ovarian syndrome, controlled ovarian stimulation, Frozen-thawed embryo transfer, Pregnancy rate.

\section{Introduction}

The syndrome of polycystic ovary (PCOS) is a common endocrine disease. This illness affects around 6.3-21.4 percent of women of reproductive age. In vitro fertilization (IVF) is one essential treatment for PCOS women in spite of the higher incidence of ovarian hyperstimulation which leads to cycle cancellation in order to prevent their high morbidity and death rates. New procedures are thus necessary to enhance clinical results [1]. We are now witnessing "freeze-all" methods that freeze the whole number of oocytes or embryos that may be used without limitation for ovarian stimulation including unfavorable hormone responsiveness reactions [2].

Early LH surge is a main cause of cycle cancellation during controlled ovarian stimulation $(\mathrm{COH})$ for women receiving IVF/ICSI therapy. The release of $\mathrm{LH}$ causes ovulation in response to rapidly increasing levels of E2 in the normal cycle, and a premature LH increase may impair IVF/ICSI egg production [3, 4]. The usage of GnRH agonists (GnRHa) and GnRH antagonists was used primarily to reduce the incidence of the early LH surge [5]. Down regulation of GnRHa promotes the synchronization of antral follicles, which results in increased procedural complexity, higher charges and higher risk of hCG triggered oval hyperstimulation syndrome (OHSS). Whilst gnRH antagonists provide fast, reversible suppression of LH without an initial flare impact, a variable percentage $(0.34 \%-38 \%)$ of GnRH patients had a premature LH outbreak, particularly older patients with decreased ovarian reserve patients $[6,7]$. New techniques with enhanced effectiveness, safety profile and user comfort are thus needed.
In 2015 Dr. Yanping Kuang of China suggested using progest-primed ovarian stimulation (PPOS), a novel ovarian stimulation system utilising progestine coupled with exogenous gonadotrophin, and ovulation co-controlled by a GnRH agonist, via 'freeze-all' methods. Instead of an increase of progesterone as in traditional protocols for ovarian stimulation, progestine is utilized as an alternative to the GnRH analogue in this novel PPOS protocol to remove early LH during the follicular phase [8]. In addition, progestin is given orally and is available readily [8]. This novel ovarian stimulation regime has shown successful prevention of a premature LH surge in cycles followed by embryo cryopreservation and does not affect the oocyte skills [8]. The selection of the right progestin is vital to the PPOS protocol's effectiveness.

The molecular structure of Dydrogesterone (DYG), comparable to natural progesterone, is extensively utilized in hormone substitution therapy, endometriosis therapeutic treatment, menstrual problems and pregnant luteal support.

Previous research utilized dydrogesterone early on the 2nd day of the cycle at the start of stimulation resulting in larger dosages of gonadotropins owing to severe hypophysical suppression.

In this research we have described how dydrogesterone may subsequently begin as a fixed PPOS regimen for moderate pituitary suppression on the 6th day of stimulation. We have developed a randomized clinical study (RCT) to investigate, and compare the cyclic properties, safety and result of pregnancy of people with PPOS. 


\section{Materials and Methods}

\subsection{Study design}

The research lasted from February 2020 to May 2021 at the Benha Fertility Center (private center).

Our research comprised a total of 76 people aged 18-39 years with PCOS who were applicants for ART therapy.

The Rotterdam criteria (2003)(9) diagnosis of PCOS included polycystic ovaries, oligo-anovulation, as well as biological or clinical indications of hyperandrogenism.

Intrauterine abnormality (submucosal fibroma, uterine polyp and intrauterine adhesion), severe endometriosis, systemic illness, and azoospermia in their spouses, have been eliminated.

The grouping took place via the disclosure of the sealed envelopes.

Each participant received 300 IU intramuscular doses ( 2 vials) of HP urinary FSH from the 2nd day of the cycle for 5 days, followed by 300 IU intramuscular doses (2 vials) of $\mathrm{HMG}$ as Meriofert 150 IU(Meriofert ${ }^{\circledR}-I B S A$ Biochimique SA) from the 6 th day of the cycle.

Women in the PPOS group got $20 \mathrm{mg}$ of dydrogesterone from the 6th day (fixed progesterone start) of their cycle (2 duphaston $10 \mathrm{mg}$ pills, one tablet every 12 hours) (douphaston, Abbott, Netherlands) and went on until trigger day

Women received in the antagonistic group 0.25 mg (Merck-Serono Germany) of cetrotide were administered subcutaneously daily on the 6th day of the cycle and continued until the trigger day.

In both categories: The ultimate triggering was accomplished by Subcutaneous Subcutaneous Injection of 2 decapeptyl ampoules of $0,1 \mathrm{mg}$ simultaneously (Ferring, Germany).

Oocytes collected 36 hours later.

\subsection{Vitrification and warming of embryos}

The cryotopic vitrification technique was used to reserve all embryos in both groups on the 3rd day following oocyte recovery.

Transfer of frozen embryos was completed 2 months later.

2.3. Preparation of the endometrium for frozenthawed embryo transmission:

The procedure of endometrial preparation in both groups was identical. The 2 nd menstrual day of the 2 nd day of the menstrual cycle, each patient took 8 $\mathrm{mg} /$ day estradiol valerate (white cycloprogenova tablets, Bayer, Germany), orally 8 days after endometrial thickness was assessed by transvaginal ultrasound and monitored every 2 days. When the thickness of the endometrial exceeded $8 \mathrm{~mm}$, oral $4 \mathrm{mg}$ estradiole valerate supplement continued (one tablet every 12 hours) and $400 \mathrm{mg}$ of pregnancy vaginal pessaries (Prontogest, Marcyrl, Egypt) began to be received twice daily until either 10 days of gestation and/or confirmed pregnancies failure. Most
HRT regimens choose to add estrogen for 2 weeks to try to imitate the NC [10].

The timing for thawing and transfer of cryopreserved embryos was dictated by the stage they were vitrified and coordinated with the time of exposure to the endometrium of progesterone.

A research in oocyte recipients revealed that the pregnancy rate was greater when progesterone supplementation was longer (i.e. the transmission of the embryo Day 3 to the 5th day of progesterone supplementation) (11).

\subsection{Pregnancy results}

Serum $\beta$ hCG $>50 \mathrm{IU} / \mathrm{L}$ two wks after ET indicated chemical pregnancy. In addition, a detection of foetal heart rhythms at 7 weeks of gestational age proved clinical pregnancy. The error was characterized as a loss of pregnancy before 20 weeks. The rate of implantation was seen as a proportion of gestational bags/embryos transplanted.

\subsection{Consideration of ethics}

This is a randomized controlled clinical intervention study authorized by the college of ethical medicine. Before this research began, all patients received signed informed permission.

\subsection{Analysis of statistics}

For data processing, data were verified, input and analysed using SPSS version 23.

Data were represented for quantitative data as a mean + standard deviation (SD) and expressed for qualitative data as frequencies and percentages. The chi-square and t-test were used in order to detect significant differences between the two groups. P < 0.05 was considered to be the threshold of significance.

\section{Results}

A total of 76 women who met the inclusion criteria were enrolled in the study as 2 groups $(\mathrm{n}=$ 38/each). The baseline characteristics were similar in both groups Table $(1,2)$.

there was statistically significant lower gonadotropin dose among antagonist group than fPPOS group (2957.8 \pm 301.9 versus $3197.4 \pm 545.9)$ respectively with increased the no. of days of stimulation in the fPPOS group. Table (3).

There was no statistically significant difference between the two studied groups regarding no. of retrieved oocytes, MII oocytes. Table (4).

Table 5 showed the comparison between the number of fertilized oocytes and number of cleaved embryos between the two studied groups which was similar.

Regarding OHSS, There was no statistically significant difference between the two group where no cases among PPOS and only one case (2.6\%) among antagonist group which had mild OHSS. Also similar results between the two groups regarding biochemical, clinical pregnancy rate and miscarriage rate. table (6). 
Table (1) Basic characteristics of the two studied groups.

\begin{tabular}{|c|c|c|c|c|}
\hline Variables & $\begin{array}{c}\text { fPPOS } \\
\text { No }=38\end{array}$ & $\begin{array}{c}\text { Antagonist } \\
\text { No }=38\end{array}$ & Test & P_Value \\
\hline Age (years) & & & $\mathrm{T}=1.3$ & 0.2 \\
\hline Mean \pm SD & $30.4 \pm 2.1$ & $29.3 \pm 4.7$ & & \\
\hline Range & $(27-34)$ & $(20-38)$ & & \\
\hline BMI $\left(\mathrm{kg} / \mathrm{m}^{2}\right.$ & & & $\mathrm{T}=0.5$ & 0.6 \\
\hline Mean \pm SD & $24.5 \pm 3.5$ & $24.2 \pm 4.1$ & & \\
\hline Range & $(19.5-29.5)$ & $(18.4-30)$ & & \\
\hline Infertility duration (years) & & & M.W 0.6 & 0.5 \\
\hline Mean \pm SD & $3.7 \pm 1.4$ & $3.9 \pm 1.6$ & & \\
\hline Range & $(19.5-5.2)$ & $(2.2-5.5)$ & & \\
\hline Infertility type & & & $\chi^{2}=0.3$ & 0.6 \\
\hline Primary & $(78.9 \%)$ & $28 \quad(73.7 \%)$ & & \\
\hline Secondary & $8 \quad(21.17 \%)$ & $10 \quad(26.3 \%)$ & & \\
\hline
\end{tabular}

M.W=Mann_Witenny U test,T-test=independent T-test.

Table (2) Baseline hormonal profile of the two studied groups.

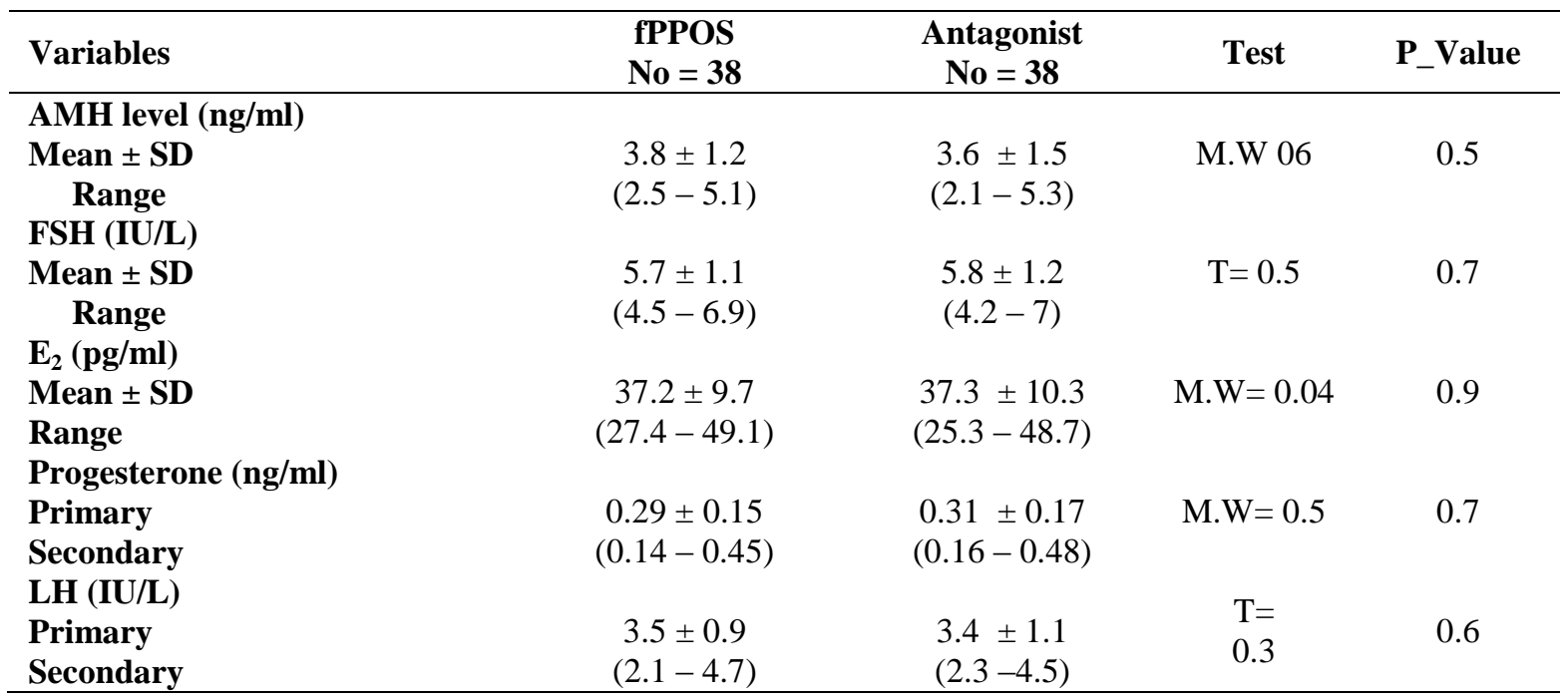

M.W=Mann_Witenny U test,T-test=independent T-test.

Table (3) Days of stimulation and total gonadotrophin dose among the two studied groups.

\begin{tabular}{|c|c|c|c|c|}
\hline Variables & $\begin{array}{c}\text { fPPOS } \\
\text { No }=38 \\
\end{array}$ & $\begin{array}{c}\text { Antagonist } \\
\text { No }=\mathbf{3 8} \\
\end{array}$ & Test & $P_{\text {_Value }}$ \\
\hline $\begin{array}{l}\text { Days of stimulation } \\
\text { Mean } \pm \text { SD } \\
\text { Range }\end{array}$ & $\begin{array}{c}10.2 \pm 1.8 \\
(8-13)\end{array}$ & $\begin{array}{c}9.5 \pm 1.1 \\
(7-11)\end{array}$ & 1.8 & 0.04 * \\
\hline $\begin{array}{l}\text { Total dose of } \\
\text { gonadotropin (IU) } \\
\text { Mean } \pm \text { SD } \\
\text { Range }\end{array}$ & $\begin{array}{l}3197.4 \pm 545.9 \\
(2400-3900)\end{array}$ & $\begin{array}{l}2957.8 \pm 301.9 \\
(2100-3300)\end{array}$ & 2.3 & $0.02 *$ \\
\hline
\end{tabular}

* Statistically significance difference.

Table (4) ovarian stimulation characteristics among the two studied group.

\begin{tabular}{|c|c|c|c|c|}
\hline Variables & $\begin{array}{c}\text { fPPOS } \\
\text { No }=38\end{array}$ & $\begin{array}{c}\text { Antagonist } \\
\text { No }=38\end{array}$ & Test & $P_{-}$Value \\
\hline $\begin{array}{l}\text { No of retrieved oocytes } \\
\text { Mean } \pm \text { SD } \\
\text { Range }\end{array}$ & $\begin{array}{c}14.7 \pm 3.1 \\
(8-19) \\
15\end{array}$ & $\begin{array}{c}14.1 \pm 3.5 \\
(6-22) \\
13\end{array}$ & $\mathrm{~T}=0.7$ & 0.4 \\
\hline
\end{tabular}




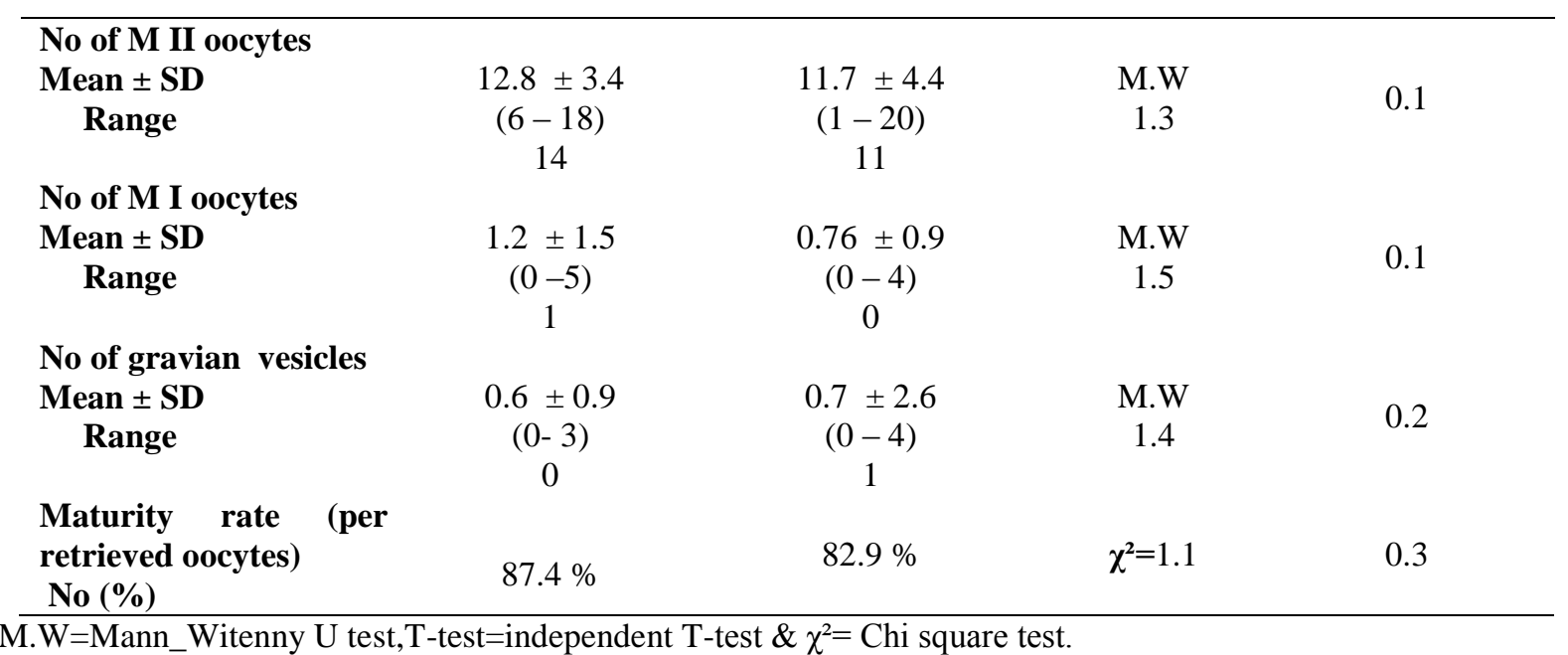

Table (5) results of ovarian stimulation and embryological outcomes among the two studied groups.

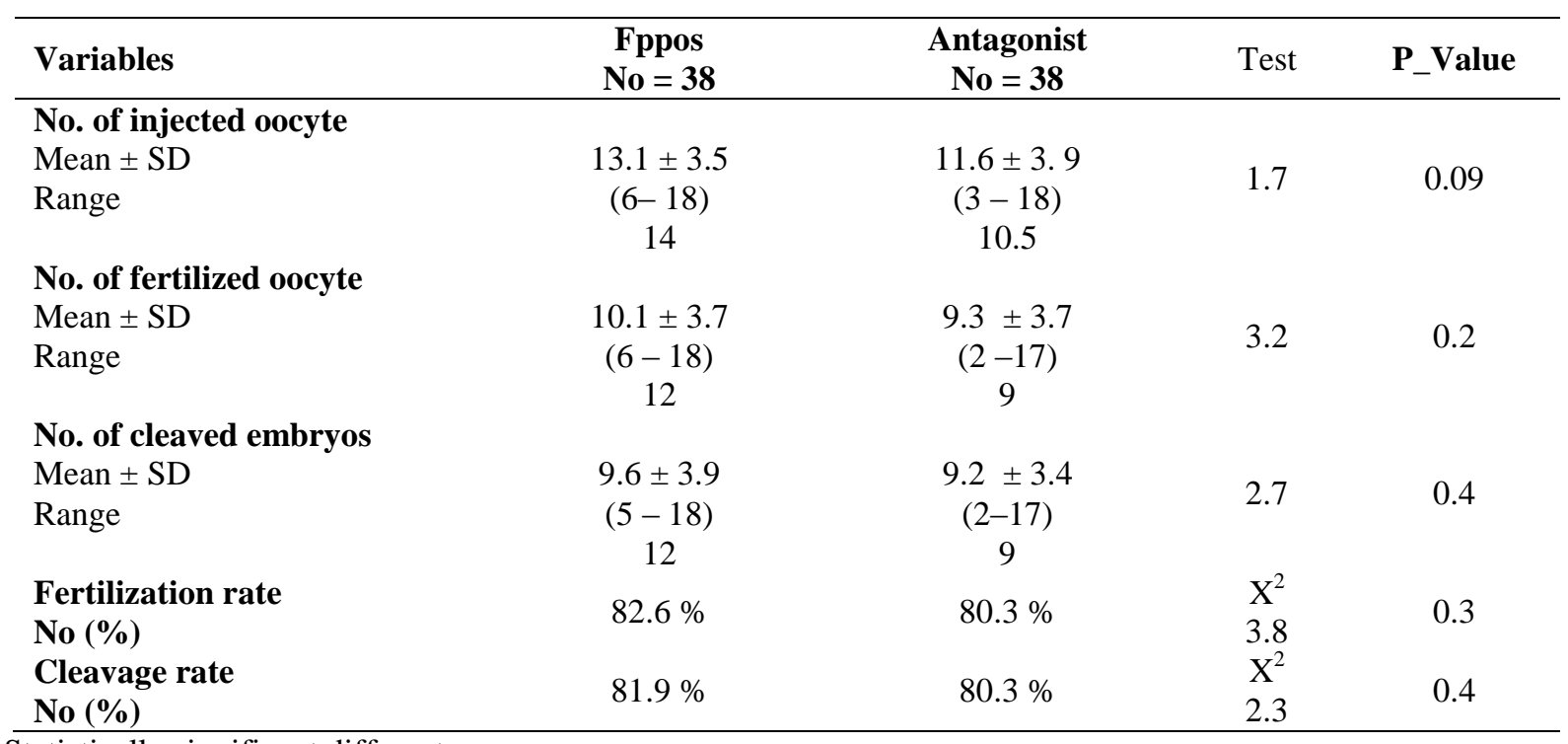

* Statistically significant different.

** Statistically highly significant.

Table (6) OHSS and Pregnancy outcomes among the two studied group.

\begin{tabular}{|c|c|c|c|c|}
\hline Variables & $\begin{array}{c}\text { Fppos } \\
\text { No }=38\end{array}$ & $\begin{array}{c}\text { Antagonist } \\
\text { No }=\mathbf{3 8}\end{array}$ & Test & $P_{-}$Value \\
\hline \multicolumn{5}{|l|}{ OHSS } \\
\hline Mild & $0 \quad(0.0 \%)$ & $1 \quad(2.6 \%)$ & & \\
\hline Moderate & $0 \quad(0.0 \%)$ & $0 \quad(0.0 \%)$ & & \\
\hline \multicolumn{5}{|c|}{ Chemical pregnancy (positive } \\
\hline B-HCG) & $9 / 38(23.7 \%)$ & $10 / 38(26.3 \%)$ & 0.07 & 0.8 \\
\hline \multicolumn{5}{|l|}{ No. $(\%)$} \\
\hline $\begin{array}{l}\text { Clinical pregnancy } \\
\text { No. }(\%)\end{array}$ & $7 / 9(77.8 \%)$ & $9 / 10(90.0 \%)$ & 0.5 & 0.4 \\
\hline $\begin{array}{l}\text { Abortion rate } \\
\text { No. }(\%)\end{array}$ & $1 / 7(14.3 \%)$ & $2 / 9(22.2 \%)$ & 0.1 & 0.7 \\
\hline
\end{tabular}

* Statistically significant difference.

\section{Discussion}

This research examined the therapeutic effectiveness of DYG in the fixed PPOS regime for PCOS. Our findings have shown that during ovarian stimulation DYG may act as an adjuvant to $\mathrm{HMG}$ when used late on 6 day to get similar oocyte recovery and viable embryo counts to the antipersonnel regimen. The baseline characteristics in both groups were comparable 
Statistically statistically significantly less in the antagonistic group than in the fPPOS group was the gonadotropin dosage $(2957.8 \pm 301.9$ vs $3197.4 \pm 545.9$ ), correspondingly a rise in stimulation days in the f PPOS group Table (3).

The figures of fertilized oocytes and the number of cleaved embryos in the two examined groups (Table 7) revealed no statistically significant differences.

The findings of this research showed that PPOS with dydroesterone was statistically comparable to the $\mathrm{GnRH}$ protocol antagonist for OHSS, where there were no instances of fPPOS and only one case (2.6\%) among antagonists who had mild OHSS. Similar findings of biochemical, clinical pregnancy and miscarriage across the two groups. Table (6).

Our findings were comparable to the design and outcomes of the Hossein Rashidi and Iwami research. There were also no significant differences between two treatment schemes in continued pregnancy (40 vs.38.1 percent) and clinical pregnancies (52.8 vs. 49.5 percent) $[12,13]$.

However, another research by Huang and our colleagues produced conflicting results. They carried out a retrospective research comparing PPOS methods with GnRH antagonists in poor IVF/ICSI respondents. They found that the PPOS protocol may increase clinical pregnancy and live birth rates in comparison to the GnRH protocol [14].

Some researchs have studied various kinds of progesterone to decrease LH during ovarian stimulation.

$\mathrm{Yu}$ and colleagues investigated the impact of dydrogesterone and medroxyprogesterone acetate (MPA) in 516 first IVF/ISCI cycles in the PPOS protocol in a forward-looking research. No early LH surge and moderate or severe OHSS occurs in individuals. Their results indicated that two groups had no significant differences and recommended that dydrogesterone may be utilized as an alternate progestin for the PPOS procedure in IVF [15].

In contrast to this research, a further investigation showed poorer reproductive results with MPA than with the antagonist of GnRH. This variation may depend on the kind, dosage and time of the progestin delivery [16].

During IVF/ICSI, Zhu and colleagues performed a two-pronged $\mathrm{COH}$ study: oral dydrogestrone + hMG (intervention group) and utroestan $+\mathrm{hMG}$ (control group). This research showed that dydrogestrone is comparable to uterine in LH-surge prevention, embryonic features and pregnancy [17].

In 2019 La Marca and Cauzzo examined papers that documented the use of ovarian stimulation exogenous progestin. The reproductive results of ovarian stimulation with progestin are comparable to those of traditional ovarian stimulation, but they felt that extensive studies were necessary to validate this [18].
A further study was conducted simultaneously during the follicular phase of hMG (150-225 IU) and MPA $(10 \mathrm{mg} / \mathrm{d})$ from cycle 3 and improve its effectiveness in terms of low incidence of premature LH infections and comparable short pregnancy results in infertile women with ordinary ovarian reserve and multifarious ovarian syndrome. They have thus acquired PPOS. The PPOS procedure breaking with the practise of relying on $\mathrm{GnRH}$ analogues (including GnRH agonists and GnRH-ants) to suppress the early LH surge, in conjunction with the entirely frozen approach, provides a novel alternative to avoid the untimely LH increase seen during the IVF. In this research, we have also shown that PPOS may successfully prevent early LH in POR patients [19, 20].

In Kuang et al. findings, hMG dosages given in the PSOP group were substantially greater in women with ordinary ovarian reserve and polycystic ovarian syndrome than those under control (short protocols), which confirms our research results. The increased total Gn units in the MPA group may be linked to the suppression of the pituitary during ovarian hyperstimulation. In women with polycystic ovarian syndrome, the incidence of OHSS was lower than that of controls.

Another research also showed that the PPOS group given gonadotropines were somewhat greater than control dosages, but the difference was not significant. It was also observed that the PPOS group had substantially greater MII oocyte, fertilization and excellent embryo rates than the anti-paralytic group $(\mathrm{p}<0.05)$. Although the clinical pregnancy and abortions between the two groups did not vary much, the clinical result of the PPOS group was somewhat better than the GnRH-ant group [14]. The fertilization and continuing pregnancy rates per transfer were substantially higher in the PPOS group than controls in Kuang et al's study ( $\mathrm{p}<0.05$ ). These findings show that PPOS procedures may enhance oocyte and embryo development potential.

Engin Turkgeldi and co-workers' research validated the novel fPPOS protocol as a flexible GnRH antagonist strategy to prevent DOR patients from premature ovulation and produces an equal proportion of metaphase-two ovocytes [21].

Another research on fPPOS revealed an increase in the number of M2 oocytes in the fPpos group in comparison with GnRH agonist group in young females with a high ovarian reserve [22]. Moreover, PPOS and GnRH agonists result in comparable M2 yields in a recent meta-analysis [23]. There is a possibility thus that beginning progesterone later in the cycle may cause milder suppression of the hypophysis, which leads to higher oocyte yields, and perhaps even PPOS. However, this potential benefit of fPPOS needs more research to be confirmed.

On the other side, another research has shown that daphastone as an assistant to FSH does not lead to comparable mature oocyte recruitment during ovarian 
stimulation. Maturity of the oocyte was primarily used for oocyte assessment. In the PPOS group the maturity rate of oocytes was substantially lower than in the antagonistic group. Furthermore, the fertilisation rate in the PPOS group was decreased. FET pregnancy in PPOS revealed a reduced rate of clinical pregnancy of $14.6 \%$ vs $29.9 \%$. The implantation rate of PPOS was also lower, albeit not statistically significant but clinically remarkable [24].

Ozgur and colleagues also found that flexiblestart MPA co-treatment was as efficient in blastocyst freeze-all IVF cycles as flexible-start $\mathrm{GnH}$-ant cotreatment in the form of LB by transmission, but MPA co-treatment OS may reduce LB per treatment and possibly cumulative LB rates because of the increased cyclic cancellation and reduced viable blastocyst numbers [25].

\section{Conclusion}

Our research showed that fixed PPOS in PCOS patients receiving IVF/ICSI therapies is a safe, efficient procedure.

\section{Conflict of Interest}

There is no Conflict of interest.

\section{References}

[1] X.Zhu, H.Ye, Y.Fu The utrogestan and hMG protocol in patients with polycystic ovarian syndrome undergoing controlled ovarian hyperstimulation during IVF/ICSI treatments. Medicine.vol.95,pp.4193-4250,2016.

[2] N.Massin New stimulation regimens: endogenous and exogenous progesterone use to block the LH surge during ovarian stimulation for IVF. Hum Reprod Update.vol.23,pp.211220,2017.

[3] JD.Neill Knobil and Neill's physiology of reproduction. 3rd ed. San Diego, CA: Elsevier.vol.15,pp.1289-301,2006.

[4] IE.Messinis Ovarian feedback, mechanism of action and possible clinical implications. Hum Reprod Update.vol.12,pp.557-71,2006.

[5] NS.Macklon, RL.Stouffer, LC.Giudice, BC. Fauser The science behind 25 years of ovarian stimulation for in vitro fertilization. Endocr Rev.vol.27,pp.170-207,2006.

[6] E.Bosch, I.Valencia, E.Escudero, J.Crespo, C.Simon, J.Remohí.Prema- ture luteinization during gonadotropin-releasing hormone antagonist cycles and its relationship with in vitro fertilization outcome. Fertil Steril.vol.80,pp.1444-9,2003.

[7] DE.Reichman, L.Zakarin, K.Chao, L.Meyer, OK.Davis, Z.Rosenwaks Diminished ovarian reserve is the predominant risk factor for gonadotropin-releasing hormone antagonist failure resulting in breakthrough luteinizing hormone surges in in vitro fertilization cycles. Fertil Steril.vol.102,pp.99-102,2014.
[8] Y.Kuang, Q.Chen, Y.Fu, Y.Wang, Q.Hong, Q. Lyu, A.Ai, Z.Shoham Medroxyprogesterone acetate is an effective oral alternative for preventing premature luteinizing hormone surges in women undergoing controlled ovarian hyperstimulation for in vitro fertilization. Fertil Steril.vol.104,pp.62-70,2015.

[9] Rotterdam ESHRE/ASRM-Sponsored PCOS consensus workshop group. Revised consensus on diagnostic criteria and long-term health risks related to polycystic ovary syndrome (PCOS). Hum Reprod.vol.19,pp.41-47,2004.

[10] P.Lutjen, A.Trounson, J.Leeton, J.Findlay, C.Wood, P.Renou The establishment and maintenance of pregnancy using in vitro fertilization and embryo donation in a patient with primary ovarian failure. Nature.vol.307,pp.174-175,1984.

[11] M-J.Escribá, J.Bellver, E.Bosch, M.Sánchez, A.Pellicer, J.Remohí Delaying the initiation of progesterone supplementation until the day of fertilization does not compromise cycle outcome in patients receiving donated oocytes: a randomized study. Fertil Steril.vol.86,pp.9297,2006 .

[12] B.Hossein Rashidi, A.Tarafdari, ST.Ghazimirsaeed, E.Shahrokh Tehraninezhad,F.Keikha,

B.Eslami.Comparison of Dydrogesterone and GnRH Antagonists for Prevention of Premature LH Surge in IVF/ICSI Cycles: A Randomized Controlled Trial. J Fam Reprod Health.vol.14(1),pp.14-20,2020.

[13] N.Iwami, M.Kawamata, N.Ozawa, T.Yamamoto, E.Watanabe, O.Moriwaka.New trial of progestinprimed ovarian stimulation using dydrogesterone versus atypical $\mathrm{GnRH}$ antagonist regimen in assisted reproductive technology. Arch Gynecol Obstet.vol.298,pp.663-71,2018.

[14]P.Huang, M.Tang, A.Qin Progestin-primed ovarian stimulation is a feasible method for poor ovarian responders undergoing in IVF/ICSI compared to a GnRH antagonist protocol: A retrospective study. J Gynecol Obstet Hum Reprod.vol.48,pp.99-102,2015.

[15] S.Yu, H.Long, HY.Chang, Y.Liu, H.Gao, J.Zhu.New application of dydrogesterone as a part of a progestin-primed ovarian stimulation protocol for IVF: Hossein Rashidi. http://jfrh.tums.ac.ir Journal of Family and Reproductive Health 3 a randomized controlled trial including 516 first IVF/ICSI cycles. Hum Reprod.vol.33,pp.229-37,2018.

[16] R.Beguería, D.García, R.Vassena, A.Rodríguez Medroxyprogesterone acetateversus ganirelix in oocyte donation: a randomized controlled trial. Hum Reprod.vol.34,pp.872-80,2019.

[17]X.Zhu, H.Ye, Y.Fu Duphaston and human menopausal gonadotropin protocol in normally 
ovulatory women undergoing controlled ovarian hyperstimulation during in vitro fertilization/intracytoplasmic sperm injection treatments in combination with embryo cryopreservation. Fertil Steri.vol.108,pp.505$12,2017$.

[18] A.La Marca, M.Capuzzo Use of progestins to inhibit spontaneous ovulation during ovarian stimulation: the beginning of a new era? Reprod Biomed Online.vol.39,pp.321-31,2019.

[19] Y.Kuang, Q.Chen, Q.Hong, Q.Lyu, A. Ai, Y.Fu, Z.Shoham Double stimulations during the follicular and luteal phases of poor responders in IVF/ ICSI programmes (Shanghai protocol). Reprod Biomed Online.vol.29,pp.684-691,2014.

[20] Y.Kuang, Q.Hong, Q.Chen, Q.Lyu, A.Ai, Y.Fu, Z.Shoham Luteal-phase ovarian stimulation is feasible for producing competent oocytes in women undergoing in vitro fertilization/intracytoplasmic sperm injection treatment, with optimal pregnancy outcomes in frozen-thawed embryo transfer cycles. Fertil Steril .vol.101,pp.105-111,2014.

[21]Engin Turkgeldi , Sule Yildiz , Sebile Guler Cekic , Bahar Shakerian, Ipek Keles \& Baris Ata Effectiveness of the flexible progestin primed ovarian stimulation protocol compared to the flexible GnRH antagonist protocol in women with decreased ovarian reserve, Human Fertility, DOI.vol.10,pp.122-133, 2020.

[22] S.Yildiz,E.Turkgeldi, B.Angun, A.Eraslan, B.Urman, \& B.Ata.Comparison of a novel flexible progestin primed ovarian stimulation protocol and the flexible gonadotropinreleasing hormone antagonist protocol for assisted reproductive technology. Fertility and Sterility.vol.112(4),pp.677-683,2019.

[23]P.Alexandru, S.Cekic, S.Yildiz, E.Turkgeldi, \& B.Ata, Progestins vs gonadotropin releasing hormone analogues for pituitary suppression during ovarian stimulation for assisted reproductive technology, a systematic review and meta-analysis. Reproductive BioMedicine Online.vol.40(6),pp.894-903.2020.

[24] M.Eftekhar, M.Hoseini, L. Saeed "Progesterone-primed ovarian stimulation in polycystic ovarian syndrome: An RCT" Int J Reprod BioMed.vol.17,pp.671-676,2019.

[25] K.Ozgur, M.Berkkanoglu, H.Bulut, L.Donmez, K.Coetzee Medroxyprogesterone acetate used in ovarian stimulation is associated with reduced mature oocyte retrieval and blastocyst development: a matched cohort study of 825 freeze-all IVF cycles. J Assist Reprod Genet. Sep.vol.37(9),pp.2337-2345.

doi: 10.1007/s10815-020-01894-4. Epub $2020 \mathrm{Jul}$ 22. PMID: 32696289; PMCID: PMC7492309, 2020 . 